\title{
Biophotonic endoscopy: a review of clinical research techniques for optical imaging and sensing of early gastrointestinal cancer
}

Authors

Institutions
Sergio Coda ${ }^{1-4}$, Peter D. Siersema ${ }^{5}$, Gordon W. H. Stamp ${ }^{2,6,7}$, Andrew V. Thillainayagam ${ }^{1-3}$

Institutions are listed at the end of article. submitted

28. September 2014 accepted after revision 7. May 2015

\section{Bibliography}

DOI http://dx.doi.org/

$10.1055 / \mathrm{s}-0034-1392513$

Published online: 8.9.2015

Endoscopy International Open

2015; 03: E380-E392

(c) Georg Thieme Verlag KC

Stuttgart · New York

E-ISSN 2196-9736

\section{Corresponding author}

Sergio Coda, MD, PhD

Imperial College London Medicine and Physics Prince Consort Road

London SW7 2AZ

United Kingdom

Fax: +44-20-75947714

s.coda@imperial.ac.uk
License terms

() (1) $\odot \circledast$
Detection, characterization, and staging constitute the fundamental elements in the endoscopic diagnosis of gastrointestinal diseases, but histology still remains the diagnostic gold standard.

New developments in endoscopic techniques may challenge histopathology in the near future. An ideal endoscopic technique should combine a wide-field, "red flag" screening technique with an optical contrast or microscopy method for characterization and staging, all simultaneously available during the procedure. In theory, biophotonic advances have the potential to unite these elements to allow in vivo "optical biopsy."

These techniques may ultimately offer the potential to increase the rates of detection of high risk lesions and the ability to target biopsies and resections, and so reduce the need for biopsy, costs, and uncertainty for patients. However, their utili-

\section{Introduction}

$\nabla$

The integration of detection, characterization, diagnosis, and staging at the time of endoscopy is still an unmet clinical need. The advent of biophotonics in endoscopy has opened new horizons and created important new opportunities for the enhanced identification and biochemical characterization of disease.

A number of biophotonic techniques are currently being investigated owing to their potential to acquire biochemical information noninvasively and to image with subcellular resolution beyond what is possible with currently available techniques; however, the published experience is limited [1].

Point spectroscopy with probes inserted through the working channels of conventional endoscopes has been able to differentiate non-neoplastic from neoplastic tissues and to examine the biochemical environment of diseased tissue. Alterations in autofluorescence spectra reflect changes in the molecular composition and biochemical en- ty and sensitivity in clinical practice must be evaluated against those of conventional histopathology.

This review describes some of the most recent applications of biophotonics in endoscopic optical imaging and metrology, along with their fundamental principles and the clinical experience that has been acquired in their deployment as tools for the endoscopist. Particular emphasis has been placed on translational label-free optical techniques, such as fluorescence spectroscopy, fluorescence lifetime imaging microscopy (FLIM), two-photon and multi-photon microscopy, second harmonic generation (SHG) and third harmonic generation (THG) imaging, optical coherence tomography (OCT), diffuse reflectance, Raman spectroscopy, and molecular imaging.

vironment of diseased tissue. Some examples of studies of gastrointestinal disease with the use of diffuse reflectance spectroscopy (DRS), which detects changes in the physical properties of cells, include the in vivo detection of dysplasia in colon polyps [2] and Barrett's esophagus [3,4], and recently, near-infrared diffuse reflectance imaging has also been applied ex vivo to surgical specimens of colon cancer [5].

A further development is fluorescence lifetime imaging microscopy, often referred to as FLIM or autofluorescence, which offers the potential to improve overall diagnostic accuracy by providing label-free quantitative contrast [6,7]. Optical coherence tomography (OCT) allows imaging at greater depths within tissue in comparison with confocal laser endomicroscopy (CLE) but does not normally supply functional information. Raman spectroscopy and coherent anti-Stokes Raman scattering (CARS) microscopy can be used to obtain highly detailed spectroscopic information but usually require long data acquisition times and cannot be readily extended to provide a 


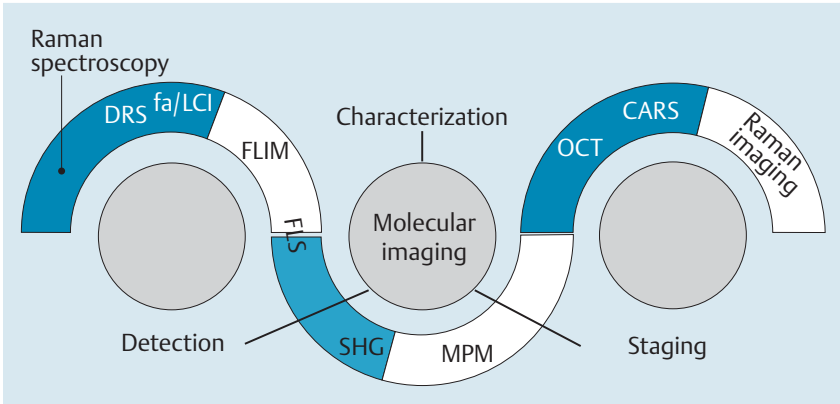

Fig. 1 Stylized Venn diagram showing predominant targets and potential relationships/overlaps between different biophotonic techniques in detecting, characterizing, and staging diseases at endoscopy. DRS, diffuse reflectance spectroscopy; fa/LCI, frequency-domain angle-resolved low coherence interferometry; FLIM, fluorescence lifetime imaging microscopy; FLS, fluorescence lifetime spectroscopy; SHG, second harmonic generation; MPM, multiphoton microscopy; OCT, optical coherence tomography; CARS, coherent anti-Stokes Raman scattering.

wide-field screening tool that can image fields of view of several centimeters. An alternative to intrinsic optical signals is the use of exogenous labels, such as labeled monoclonal antibodies against the carcinoembryonic antigen (CEA), or the epidermal growth factor receptor (EGFR), or photosensitizers administered topically. These markers can then be imaged with a range of instruments, such as confocal endomicroscopy or prototype fluorescence endoscopes [8-10]. These techniques are generally referred as to molecular imaging. However, most of these diagnostic applications are still in the early stage of development.

Biophotonics is a relatively novel, interdisciplinary science that uses light-based technology to image, detect, and characterize the interplay between light and biological materials, including cells and tissues in living organisms.

When light reaches the surface of a tissue, it may either be directly reflected or enter the tissue. Light is scattered within the tissue owing to small differences in the refractive index caused by cell nuclei and cell organelles. It may then exit the tissue surface as diffusely reflected light or be absorbed by molecules within the tissue. If the light is absorbed, it may be converted to fluorescence. This fluorescence light is again scattered within the tissue and may be reabsorbed by another molecule or may exit the tissue.

Thus, based on this complex interaction, biophotonic techniques can be classified mainly as scattering or fluorescence-based. This classification also takes into account the excitation wavelength used to actualize the process (ultraviolet- visible vs. near-infrared spectrum).

It is difficult at present to classify each method according to its potential clinical use and according to what diseases it is best applied because different modalities might be used for one or all elements of diagnosis and be applied to a variety of different conditions. Although individual techniques aim to prove useful for a specific aspect of the diagnostic process, there is some cluster overlap between the elements, as shown in the stylized Venn diagram in 0 Fig. 1.

The most appropriate and useful method for classifying biophotonic endoscopic techniques is probably according to their ability to provide functional and biochemical information and/or to enhance spatial resolution. In order to present some of the relevant features of each technique in a graphic form, we have placed each modality on a two-dimensional grid where the axes are spatial

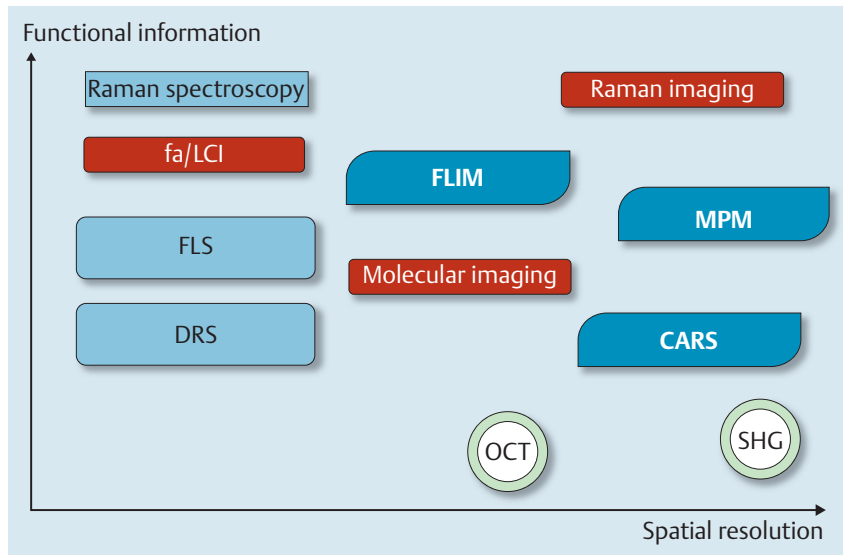

Fig. 2 Two-dimensional grid-based classification of biophotonic techniques according to their ability to provide functional information and/or to enhance spatial resolution. $\mathrm{fa} / \mathrm{LCl}$, frequency-domain angle-resolved low coherence interferometry; FLS, fluorescence lifetime spectroscopy; DRS, diffuse reflectance spectroscopy; FLIM, fluorescence lifetime imaging microscopy; MPM, multiphoton microscopy; CARS, coherent anti-Stokes Raman scattering; OCT, optical coherence tomography; SHG, second harmonic generation.

resolution and amount of functional information ( $\bullet$ Fig.2). However, it should be noted that with further development, some techniques, such as FLIM, molecular imaging, and OCT, may be able to offer both functions either individually or combined. Ultimately, in terms of the propagation of light into tissue and axial resolution, they can be further classified as up to or beyond $1000 \mu \mathrm{m}$ ( Table 1$)$.

The following sections review the fundamental principles of and clinical experience with the most recent translational biophotonic modalities, which may offer improved image resolution, contrast, and tissue penetration and may report on biochemical and molecular changes beyond the discrimination of existing endoscopic methods.

A glossary of key terms and definitions appears in $\bullet$ Table 2 to clarify the terminology used throughout this review.

\section{Techniques providing functional/biochemical information \\ $\nabla$}

Fluorescence spectroscopy and lifetime imaging

Fluorescence is defined as the emission of light by a medium (e.g., tissue) that has absorbed light of a different wavelength. In most cases, the emitted light has a longer wavelength, and therefore a lower energy level, than the absorbed light. Fluorescence can use endogenous (autofluorescence) or exogenous fluorophores (i.e., fluorescein) to achieve contrast and distinguish between normal and diseased tissue, based on specific molecular characteristics of the tissue. Particularly, autofluorescence is generated by intra- and extracellular endogenous fluorophores, such as collagen, elastin, and pyridine nucleotides, and the distribution of these fluorophores can be different in normal and diseased tissue.

However, intensity-based autofluorescence contrast is often not sufficiently specific because it is difficult to make quantitative intensity measurements. Therefore, when the autofluorescence of biological tissue is studied, the molecular composition is often unknown, and so tissue autofluorescence can present a complex 
Table 1 Summary of emerging biophotonic techniques in endoscopy.

\begin{tabular}{|c|c|c|c|c|c|c|c|c|}
\hline Technique & $\begin{array}{l}\text { Func- } \\
\text { tion }\end{array}$ & $\begin{array}{l}\text { Morphol- } \\
\text { ogy }\end{array}$ & $\begin{array}{l}\text { Cross-sec- } \\
\text { tionable }\end{array}$ & $\begin{array}{l}\text { Maximum } \\
\text { depth, } \mu \mathrm{m}\end{array}$ & Excitation, $\lambda$ & $\begin{array}{l}\text { Acquisition } \\
\text { time, } s\end{array}$ & $\begin{array}{l}\text { Clinical } \\
\text { experience }\end{array}$ & References \\
\hline OCT & N & Y & Y & 4000 & Near-IR & 1.5 & Ex vivo, in vivo & $48-62$ \\
\hline FLIM & $\mathrm{Y}$ & Y & Y (confocal) & $<100$ & UV-blue & $20-120$ & Ex vivo, in vivo & $1,6,7,19,78,82$ \\
\hline FLS & $\mathrm{Y}$ & $\mathrm{N}$ & $\mathrm{N}$ & $<100$ & UV-blue & $<15$ & Ex vivo, in vivo & $6,14,15$ \\
\hline $\begin{array}{l}\text { Raman } \\
\text { spectroscopy }\end{array}$ & $\mathrm{Y}$ & $\mathrm{N}$ & $\mathrm{N}$ & $>1000$ & Near-IR & $0.1-5$ & Ex vivo, in vivo & $26-28$ \\
\hline Raman imaging & Y & Y & $\mathrm{N}$ & $<100$ & Near-IR & $>1800$ & Ex vivo & 72 \\
\hline DRS & N & N & N & $<1000$ & White light & 0.5 & Ex vivo, in vivo & $2,3,5,34-38$ \\
\hline MPM & Y & Y & Y & $<2000$ & Near-IR & 1 & Ex vivo & $66-70$ \\
\hline SHG & Y & Y & Y & $100-300$ & Near-IR & 1 & Ex vivo & 71 \\
\hline CARS & Y & Y & N & $>1000$ & Near-IR & 5.4 & Ex vivo & $72-74$ \\
\hline $\mathrm{fa} / \mathrm{LCl}$ & N & Y & $\mathrm{N}$ & $<150$ & Near-IR & $<1$ & Ex vivo & 39,40 \\
\hline Molecular imaging & Y & Y & $Y($ confocal) & $>1000$ & Blue, near-IR ${ }^{1}$ & 2 & Ex vivo & $8-10,41-47$ \\
\hline
\end{tabular}

OCT, optical coherence tomography; FLIM, fluorescence lifetime imaging microscopy; FLS, fluorescence lifetime spectroscopy; DRS, diffuse reflectance spectroscopy; MPM, multiphoton microscopy; SHG, second harmonic generation; fa/LCl, frequency-domain angle-resolved low coherence interferometry; near-IR , near-infrared.

1 Dependent on fluorescent label.

2 Dependent on technique used.

Table 2 Glossary of key terms and definitions in biophotonics.

\begin{tabular}{|ll|}
\hline Biophotonics & $\begin{array}{l}\text { Novel interdisciplinary science using light-based } \\
\text { technology to image, detect, and characterize the in- } \\
\text { terplay between light (photons) and biological mate- } \\
\text { rials, including cells and tissues in living organisms. }\end{array}$ \\
\hline Biophotonic & Of or pertaining to biophotonics. \\
\hline Label-free & $\begin{array}{l}\text { Defines detection methods not requiring the admin- } \\
\text { istration of external agents to achieve contrast. }\end{array}$ \\
\hline Metrology & $\begin{array}{l}\text { Science studying, defining, and investigating meas- } \\
\text { urements (e.g., spectra) but not imaging phenom- } \\
\text { ena. }\end{array}$ \\
\hline Spectroscopy & $\begin{array}{l}\text { Science investigating and measuring spectra resulting } \\
\text { from the interaction of light and matter (e. g., biolo- } \\
\text { gical tissue). }\end{array}$ \\
\hline Fluorescent & $\begin{array}{l}\text { External molecules (e. g., contrast agents or drugs) } \\
\text { capable of absorbing light of a specific wavelength } \\
\text { probes }\end{array}$ \\
\hline veluomitting light of a different, typically longer wa- \\
velength to produce visible and measurable fluores- \\
cence.
\end{tabular}

signal resulting from an unknown number of endogenous fluorophores present in unknown relative concentrations. The interpretation of autofluorescence data is therefore highly challenging, typically requiring a priori knowledge of tissue structure and/or physiologic function to yield quantitative information.

\section{Fluorescence lifetime}

Fluorophores are molecules within tissue that spontaneously emit light when illuminated by light at appropriate wavelengths. When these molecules are illuminated, they can absorb energy from the incident photons, and an electron within the molecule may enter an excited state. Following light absorption, a fluorophore will remain in an excited electronic energy state for a very short period of time before returning to its ground state via the emission of fluorescent light, in a manner somewhat similar to the radioactive decay of an unstable atom.

A fluorophore in the excited electronic state may also return to the ground state by a number of nonradiative processes (i.e., without the emission of fluorescence). Thus, fluorescent light from a sample does not instantaneously cease the moment that the excitation light is extinguished; rather, it decays away over a period of nanoseconds as excited fluorophore molecules in the sample return to their ground state. This period is referred to as fluorescence lifetime $(\tau)$, and the energy transfer is schematically illustrated with the classic Jablonski diagram ( Fig. 3a). The key point is that different fluorophores decay at different rates, and this parameter can be mapped at every pixel in an image in order to produce a fluorescence lifetime image ( $\bullet$ Fig.3b). Typical fluorescence lifetimes are of the order of picoseconds $\left(10^{-12} \mathrm{sec}\right.$ onds) to nanoseconds ( $10^{-9}$ seconds).

FLIM is a powerful means to map the decay rate of fluorescence emitted from tissue samples, providing quantitative contrast. It can be achieved for a variety of fluorescence imaging instruments, including wide-field imaging, confocal microscopy, and two-photon microscopy.

Optical fiber probe-based fluorescence lifetime spectroscopy (FLS) can resolve the fluorescence emission spectrum and fluorescence decay rate to provide a wealth of label-free (i.e., no need for external contrast agents) information from biological tissue. The lifetime of a fluorophore depends on the rate of decay from the excited electronic state back to the ground state by both radiative $(\Gamma)$ and nonradiative $(\mathrm{K})$ means $(\bullet$ Eq. $\mathbf{1})$ :

$\tau=\frac{1}{\Gamma+\mathrm{K}}$

The radiative decay rate $(\Gamma)$ is essentially a function of the fluorophore and is relatively unaffected by its surrounding environment. Conversely, the nonradiative decay rate $(\mathrm{K})$ depends on the local physicochemical environment of the fluorophore. It may be sensitive to a number of different parameters, such as viscosity, temperature, $\mathrm{pH}$, and oxygen concentration, as all these factors may influence the intermolecular events that account for the nonradiative transition of an excited fluorophore to its ground state. Therefore, fluorescence lifetime can be influenced by, and so can be used as an indicator of, a certain fluorophore's local environment. Thus, the measurement of temporally resolved fluorescence affords the possibility of obtaining functional as well as structural information.

Another important characteristic of the fluorescence lifetime is that it is an inherently ratiometric quantity because it depends 


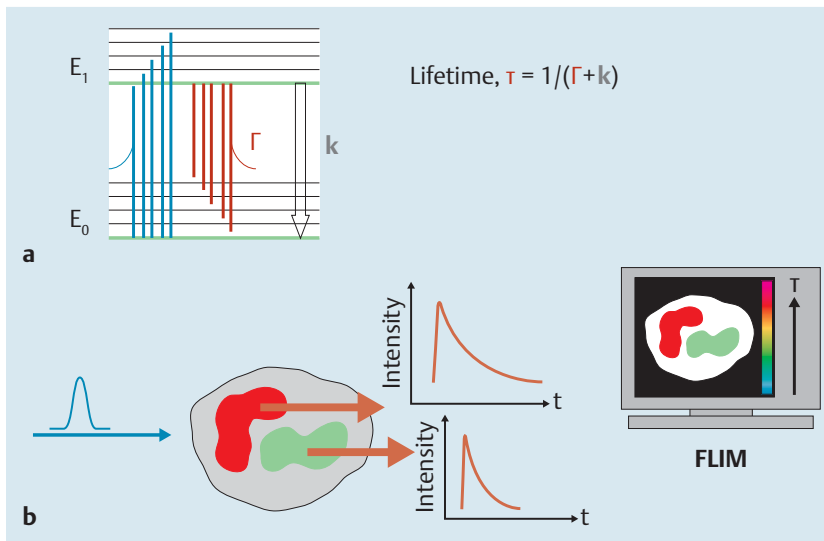

Fig. 3 a Jablonski diagram showing electronic energy levels of ground $\left(E_{0}\right)$ and excited $\left(E_{1}\right)$ states and both radiative $(\Gamma)$ and nonradiative $(\kappa)$ decay pathways in a fluorescent molecule. $\mathbf{b}$ After a short pulse of light, fluorescence emitted from the sample decays away over a period of nanoseconds as excited molecules return to their ground state. Fluorescence lifetime imaging microscopy (FLIM) produces images by using the fluorescence lifetime determined at each pixel to provide image contrast, displayed through a false-color scale, between tissues or different components within the tissue that have different fluorescence decays.

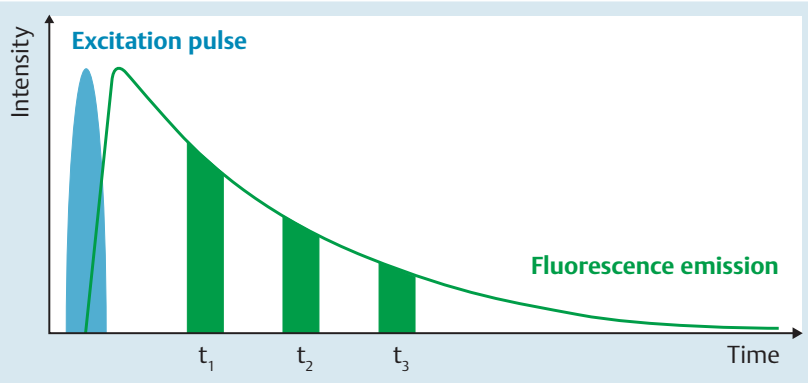

Fig. 4 The principle of time-gated detection for fluorescence lifetime imaging microscopy (FLIM). The excitation light pulse is shown in purple, with the fluorescence emission in green. Three detection time gates $\left(t_{1}, t_{2}\right.$, and $t_{3}$ ) are also shown.

on the intensity decay as a function of time and not on the absolute intensity. As such, unlike measurements with the intensity signal alone (e.g., standard autofluorescence), measurements of the fluorescence lifetime do not suffer from uncertainties due to variations in fluorophore concentration or excitation power. For this reason, considerable research efforts have been directed toward the development of instruments for time-resolved fluorescence spectroscopy and imaging. Most importantly, the fluores- cence lifetime has been shown to help discriminate between healthy and diseased tissue $[7,11-17]$.

\section{Measurement of fluorescence lifetimes}

There are two main ways of performing FLIM: time domain and frequency domain.

Fluorescence lifetime in the time domain is measured by recording the intensity decay of fluorescence after excitation with a very short pulse of light. It can be done either by time gating (৫ Fig.4), as is used in wide-field microscopes, or by time-correlated single-photon counting (TCSPC), as is used in confocal microscopes and spectroscopy systems ( $\bullet$ Fig.5). Time domain measurements are the most intuitive because they involve direct measurement of the decay of fluorescence intensity over time. Frequency domain FLIM uses sinusoidally varying excitation light. The resultant fluorescence is then also sinusoidally modulated, but with a reduction in the modulation depth (and average intensity) and a shift in phase ( $\bullet$ Fig.6).

\section{Instrument response function}

When a fluorescence decay measurement is performed, it should be noted that the signal received depends not only on the sample being analyzed but also on the response of the measuring system. These factors combine to form an instrument response function that must be taken into account when fitting fluorescence decays. The method used to obtain the instrument response function depends on the system being used, but it usually involves measuring the time response of a fluorophore with a short lifetime (e.g., erythrosin B, daspi) or a scatterer (e.g., Ludox).

\section{Displaying fluorescence lifetime imaging microscopy (FLIM) images}

Once a decay model has been fitted to every pixel within an image, it is then necessary to display the data in an accessible and informative manner. The most straightforward way to do this is to make a false-color image. In this manner an 8-bit color image is formed in which typically blue represents shorter lifetimes and red is for longer lifetimes, corresponding to a lifetime scale specified by the user. This display strategy takes no account of intensity, and therefore some important structural information may be lost; this may be remedied by merging the FLIM map with the integrated intensity image. In this way, the structural information is maintained, and pixels with low signals, which may have less accurate lifetime fits, are given less emphasis. Images are merged in LabVIEW (National Instruments, Austin, Texas, USA). An example of a FLIM map, intensity image, and merge is shown in $\bullet$ Fig. 7.

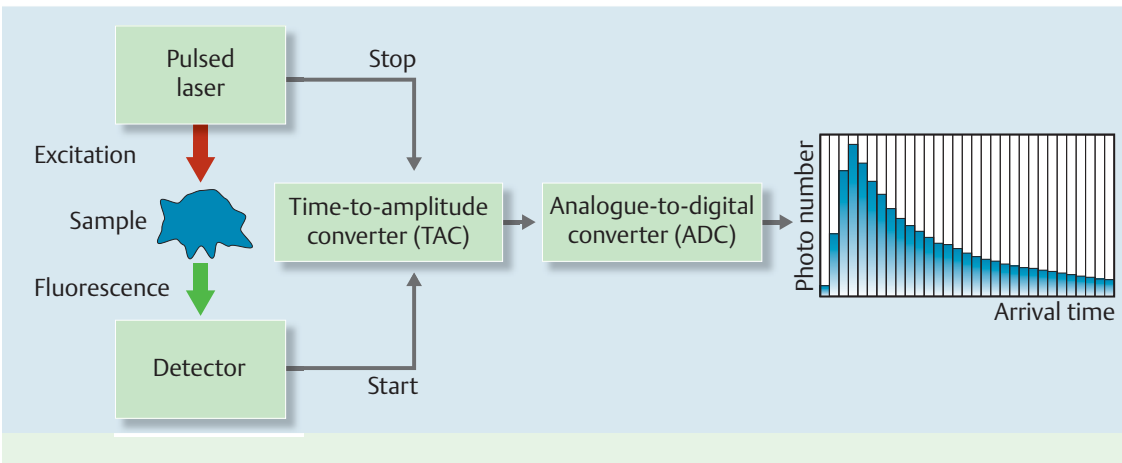

Fig. 5 Schematic diagram of the time-correlated single-photon counting (TCSPC) acquisition procedure. When a fluorescence photon is detected, typically by a photomultiplier tube, an electronic signal is sent to a time-to-amplitude converter (TAC), which starts to linearly charge a capacitor; the charging is subsequently stopped when the laser next emits a light pulse. The charge on the capacitor is then fed into an analogue-to-digital converter (ADC) and the time recorded in memory. Repetition of this process for many detected photons allows a histogram of photon arrival times to be built up, and decay models can then be fitted to these data. 


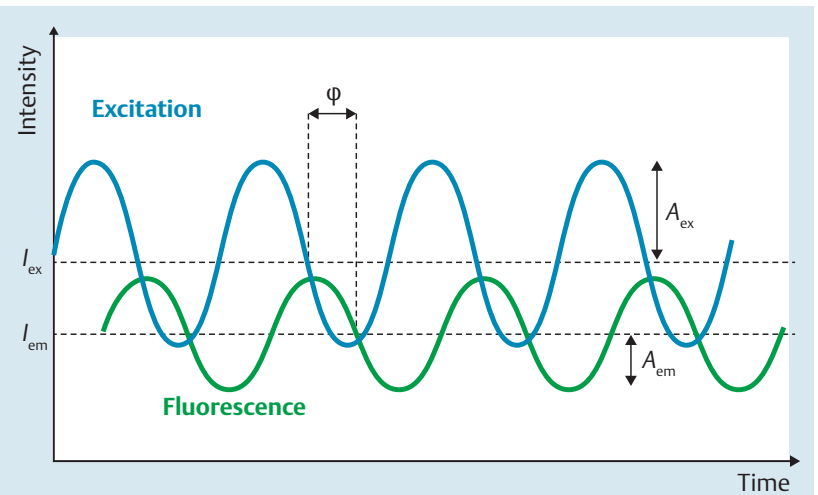

Fig. 6 Phase shift and demodulation seen in frequency domain fluorescence lifetime imaging microscopy (FLIM) during excitation with sinusoidally modulated light. The phase shift $(\varphi)$ is labeled, as are the amplitudes of the excitation light $\left(A_{\mathrm{ex}}\right)$ and emitted light $\left(A_{\mathrm{em}}\right)$ and the respective average intensities $\left(l_{\mathrm{ex}}\right.$ and $\left.l_{\mathrm{em}}\right)$.

\section{Clinical experience}

Time-resolved autofluorescence spectroscopy in the gastrointestinal tract was pioneered by Mycek et al. [11]; this in vivo study of 24 polyps (13 adenomas, 11 non-adenomas) from 17 patients used an excitation wavelength of $337 \mathrm{~nm}$ and detection in the range 530 to $570 \mathrm{~nm}$ and demonstrated shorter average lifetimes for neoplastic polyps (adenomas) than for non-neoplastic polyps ( $9300 \pm 400$ vs. $10500 \pm 700$ picoseconds). This was the first study in the colon that demonstrated differences in fluorescence lifetime decay between nondysplastic and dysplastic tissue. The shift in fluorescence lifetime made it possible to distinguish between the two tissue types investigated with a sensitivity of $85 \%$, a specificity of $91 \%$, a positive predictive value of $92 \%$, and a negative predictive value of $83 \%$.

In work by Li et al. in ex vivo tissue, longer autofluorescence lifetimes were found in cancerous colonic tissues than in normal colon (18450 vs. 4320 picoseconds) with excitation at $397 \mathrm{~nm}$ and detection at $635 \mathrm{~nm}$. This was attributed to the contribution of protoporphyrin IX (PpIX), which is known both to accumulate in neoplasia and to have a long fluorescence lifetime [18].

With the use of wide-field FLIM, statistically significant autofluorescence lifetime contrast was observed between cancerous and healthy colon tissue in a series of 16 unstained surgical specimens of colon cancer. In this study, the mean lifetime of autofluorescence from neoplastic tissue induced by 355-nm ultraviolet excitation was longer than autofluorescence from surrounding non-neoplastic tissue $(3700 \pm 1100$ vs. $3200 \pm 420$ picose- conds) [7]. Autofluorescence lifetime contrast between cancerous and healthy tissue was also shown in a specimen of unfixed ex vivo liver containing metastatic colorectal carcinoma and in an unfixed pancreas containing an area of pancreatic adenocarcinoma.

Preliminary data from our group have shown a statistically significant decrease in 435-nm excited, spectrally averaged mean fluorescence lifetime (mean $\Delta \tau=-570 \pm 740$ picoseconds, $P=$ 0.021 ) in a comparison between normal tissue and neoplastic colonic polyps obtained from 12 patients undergoing colonoscopy as part of their clinical investigation. The shift in spectrally averaged mean fluorescence lifetime was found to be correlated with the shift in the emission spectrum $\left(r^{2}=0.71\right)$. Although the difference in the spectrally averaged mean lifetime shift between neoplastic and non-neoplastic polyps was not found to be statistically significant, if the changes in mean autofluorescence lifetime and emission wavelength of 435-nm excited fluorescence are combined, then a discrimination of neoplastic from non-neoplastic polyps with a sensitivity of $75 \%$ and a specificity of $83 \%$ is achieved. This study was undertaken with a compact, clinically compatible endoscopic fiberoptic coupled spectrofluorometer ( Fig. 8) using all-solid-state picosecond diode lasers to provide simultaneous measurements of time-resolved fluorescence spectra with two excitation wavelengths (375 and $435 \mathrm{~nm}$ ) [15].

A current prospective in vivo study with the same FLS system is under way to determine if the trends observed ex vivo are confirmed. Initial analysis has indicated that at 435-nm excitation, polyps exhibit mean fluorescence lifetime values shorter than those of normal tissue, and although no diagnostically significant differences were observed in the signals obtained in vivo, these data are consistent with our ex vivo measurements. When different regions of endoscopically and histologically normal colon (rectum, left and right colon) were compared with respect to their spectrally averaged normalized emission spectrum and mean fluorescence lifetime, statistically significant differences were obtained for $375-\mathrm{nm}$ excitation $(P=0.0017)$, with the greatest difference observed between right and left colon $(P=0.001)$. Pfefer et al. [12] used excitation wavelengths of $337 \mathrm{~nm}$ and 400 $\mathrm{nm}$ and compared the measurement of fluorescence emission spectra with fluorescence decay profiles acquired at $550 \mathrm{~nm}$ in vivo in 37 patients ( 17 at $337 \mathrm{~nm}$ and 20 at $400 \mathrm{~nm}$ ) undergoing routine endoscopic surveillance for Barrett's esophagus. In this study, time-resolved fluorescence was unable to achieve sensitivity and specificity values above $60 \%$ for discriminating high risk (carcinoma, high grade dysplasia) from low risk (low grade dysplasia, no dysplasia, or indefinite for dysplasia) tissue, whereas moderate overall accuracy was achieved with steady-state fluor-
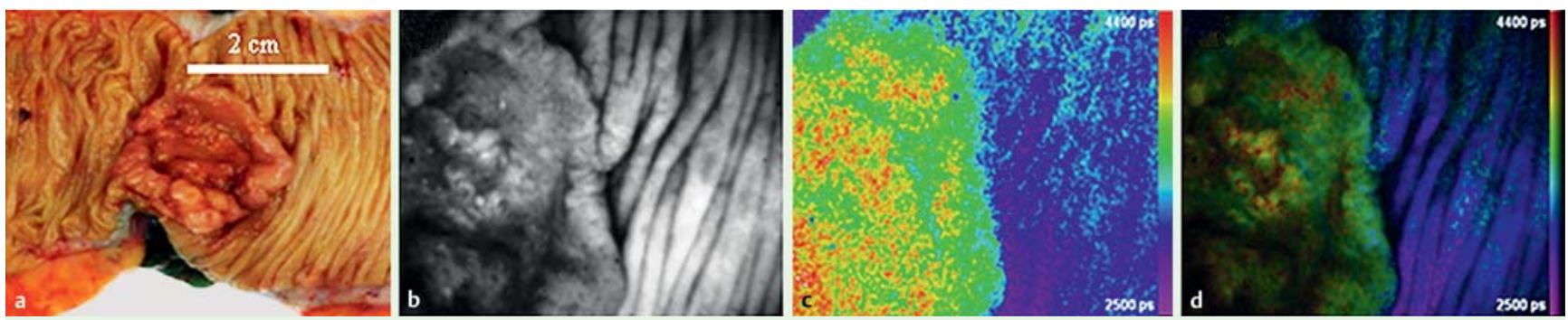

Fig. 7 Matched white light, fluorescence intensity, and fluorescence lifetime imaging microscopy (FLIM) images of a fresh hemicolectomy specimen containing a moderately differentiated colonic adenocarcinoma. a White light image. b Fluorescence integrated intensity image. c False-color FLIM map. d FLIM merge image showing clear visual contrast between areas of normal and malignant tissue. (Adapted and reproduced with permission from McGinty J, Galletly NP, Dunsby C et al. Wide-field fluorescence lifetime imaging of cancer. Biomed Opt Express 2010; 1: 627-640 [7].) 


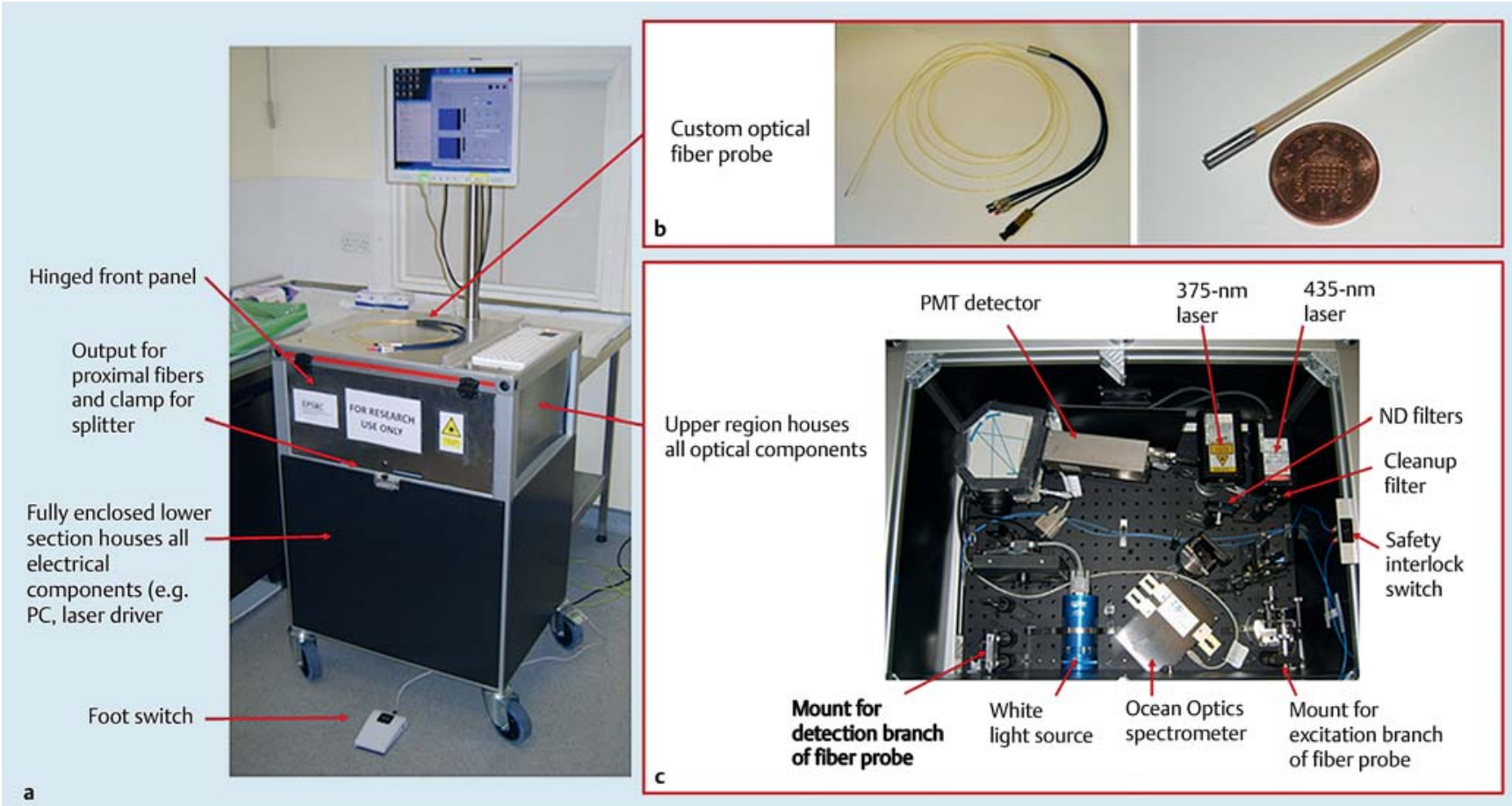

Fig. 8 a Annotated photograph of the transportable trolley that houses the entire endoscopic spectrofluorometer, showing some important features of the fiberoptic fluorescence lifetime spectroscopy (FLS) system developed by the Photonics Group at Imperial College London. b The custom-built fiber probe for research use in gastrointestinal endoscopy (FiberTech $\mathrm{GmbH}$, Berlin, Germany). c Annotated high-angle photograph illustrating the optical arrangement of the time-resolved spectrofluorometer with the lid removed. PMT, photomultiplier tube; ND, neutral density. (Source of acquisition: Endoscopy Unit, Charing Cross Hospital, Imperial College Healthcare NHS Trust, London, United Kingdom.)

escence only (74\% sensitivity, $67 \%$ specificity and $74 \%$ sensitivity, $85 \%$ specificity for 337-nm and 400-nm excitation, respectively). Recently, Sun et al. developed and applied in vivo a clinically compatible FLIM system consisting of a rigid fiber-bundle endoscope proving a field of view of $4 \mathrm{~mm}$. Intraoperative imaging of head and neck squamous cell carcinoma was performed in 10 patients at 337-nm excitation and fluorescence collected in the 435- to 485-nm range with the intent of facilitating tumor margin demarcation. Head and neck squamous cell carcinoma exhibited a shorter average lifetime ( $1210 \pm 40$ picoseconds) than the surrounding normal tissue (1490 \pm 60 picoseconds) [19].

\section{Raman spectroscopy}

Raman spectroscopy has been used to probe the biochemical signatures of a multitude of biological molecules. The Raman effect results from energy exchange between incident light and scattering molecules within tissue. It is also referred to as inelastic scattering because Raman scattered light has less energy than the incident light. Raman signals have typical narrow peaks that are generally associated with the vibration of a particular chemical bond in a molecule. As the bond stretches and shrinks, a shift in frequency, which is characteristic of a particular molecule, is indicated in the spectrum. Therefore, Raman spectroscopy can provide highly specific molecular fingerprint information that is chemically sensitive and label-free. Raman spectroscopy can be integrated with confocal microscopy for high spatial resolution. However, Raman signals are usually weak (cross section typically 1000 times lower than fluorescence), and images require high laser power (typically $>10 \mathrm{~mW}$ ), ultrasensitive detectors, and long acquisition times ( $>30$ minutes), which are unsuitable for in vivo clinical use.

\section{Clinical experience}

The feasibility of in vivo Raman spectroscopy for use in gastrointestinal endoscopy was first demonstrated in 2000 [20]. Raman spectra were obtained from various organs in the gastrointestinal tract, including the esophagus and colon, during endoscopy with a custom-built fiberoptic near-infrared Raman spectroscopic system at 785-nm excitation, but because of the limited set of data, only subtle differences were observed between normal and pathologic states. Later on, distinctive in vivo Raman spectra were shown by the same group in a comparison between 10 adenomas and 9 hyperplastic colon polyps with 95\% accuracy [21]. Principal component and linear discriminant analyses were used to develop a diagnostic prediction model for classifying colon polyps based on their spectral emission. Raman spectra were also analyzed from 20 hyperplastic and 34 adenomatous polyps, with accuracy (93\%) similar to that of the in vivo measurements. Microscopy-based Raman spectroscopy at 830-nm excitation was used to classify and discriminate between normal and pathologic states, with a particular focus on early epithelial malignancies, in a variety of organs, including larynx, esophagus, and bladder [22]. Tissues were obtained during endoscopic or surgical procedures and immediately snap-frozen. The histological assessment preceded the spectral measurements, so that only samples with clearly defined pathologies were measured. In the esophagus study, Raman spectra were measured from 50 samples classified in eight different tissue states: normal squamous epithelium, cardiac Barrett's esophagus, fundic Barrett's esophagus, intestinal metaplasia, high grade dysplasia, adenocarcinoma, squamous dysplasia, and squamous cell carcinoma. No agreement was achieved on samples with low grade dysplasia, so this group was excluded from the analysis. Multivariate linear discriminant anal- 
ysis was performed on the eight groups, and when the spectra were combined into three clinically significant groups (normal, Barrett's esophagus, and Barrett's esophagus neoplasia), 93.2\% and $88.6 \%$ of the cases were correctly predicted by the threeand eight-group models, respectively. The development of a custom-built confocal Raman fiberoptic probe for endoscopic use was then presented by the same group [23]. Raman spectra were obtained from 58 esophageal biopsy specimens, and the probe performance was compared with that of a conventional spectrometer at both 2 - and 10-second acquisition times.

Subsequent ex vivo work with the use of this probe at $830-\mathrm{nm}$ excitation in 72 esophageal samples from 35 patients, with acquisition periods of 10 and 2 seconds, achieved a $73 \%$ overall correct classification in spectrally separating histological diagnoses into three clinically significant groups: normal, low risk (Barrett's esophagus), and high risk (dysplasia and cancer) [24]. Recent ex vivo work with the probe in 298 fresh and 39 snap-frozen esophageal specimens, collected during surgery or endoscopy from 28 patients, confirmed the ability of this system to discriminate between Barrett's esophagus and neoplasia with high levels of sensitivity and specificity ( $88 \%$ and $87 \%$, respectively) [25].

A follow-up study from the same group, of a larger number (673) of ex vivo esophageal samples collected surgically and endoscopically from 62 patients who had Barrett's esophagus with a novel endoscopic Raman probe, has confirmed the preliminary findings, with similar sensitivity (86\%) and specificity (88\%) for detecting high grade dysplasia and adenocarcinoma. Interestingly, the potential for combination with narrow-band imaging was also demonstrated [26].

Using a fiberoptic Raman endoscopy system under trimodal imaging guidance (white light endoscopy [WLE]+ narrow-band imaging $[\mathrm{NBI}]+$ autofluorescence imaging $[\mathrm{AFI}])$, an in vivo pilot study was recently conducted in 27 patients with suspected esophageal cancer [27]. Non-negativity constrained least squares minimization (NNCLSM) biomolecular modeling and linear discriminant analysis were used to develop a diagnostic algorithm for separating normal esophageal mucosa from cancer with $97 \%$ sensitivity and $95 \%$ specificity.

Impressively, the full potential of moving Raman spectroscopy into real time during routine endoscopy has recently been realized in the context of a national gastric cancer screening program in Singapore, where a large number of Raman spectra (2465 normal and 283 gastric cancer) were acquired at 785-nm excitation from 305 patients [28]. The algorithm analysis developed had modest overall diagnostic accuracy, but most importantly, the study showed that real-time acquisition per measurement $\left(<0.5\right.$ second) with a reasonably low power $\left(1.5 \mathrm{~W} / \mathrm{cm}^{2}\right)$ can be achieved, which is critical for in vivo clinical applications.

Previous in vivo work published by the same group, combining near-infrared autofluorescence and Raman spectroscopy, revealed significant differences in both the spectral intensities and Raman peaks of different colonic tissues, including normal colon and hyperplastic and adenomatous polyps [29]. The intensities of the adenomatous polyps were significantly lower than those of the hyperplastic polyps and normal tissue $(P<0.001)$. The spectral shapes from different types of colonic tissues showed different decreasing trends from 810 to $1050 \mathrm{~nm}$. Based on previous experience [30], the authors assumed porphyrins to be the major endogenous fluorophores responsible for near-infrared autofluorescence emission in tissue, particularly in proliferative lesions [31]. Increased contents of porphyrins for the adenomatous polyps in comparison with the hyperplastic polyps and normal colonic tissue were found with the use of NNCLSM biochemical modeling.

As anticipated in pioneering studies [21,32], with further development this technique has the potential to distinguish adenomas from hyperplastic or inflammatory colonic polyps, and low grade from high grade dysplasia within Barrett's esophagus, lesions whose diagnosis still presents major challenges in gastrointestinal pathology.

\section{Diffuse reflectance spectroscopy}

DRS, also known as elastic scattering spectroscopy (ESS), is used to study the diffuse component of reflected light that has been scattered multiple times within the tissue sample to be investigated. A white light source, rather than a laser, is typically used to illuminate the sample, and the reflected light is collected and transmitted by optical fibers to the analyzing spectrometer. Delivery and collection fibers are usually separated by a set distance in order to allow the spectrometer to preferentially detect diffuse (rather than specular) reflections. The resulting data then consist of the diffuse reflectance of the sample as a function of wavelength.

Because the propagation of light through a biological specimen depends on its intrinsic absorptive and scattering properties, the light elastically scattered can report on the architectural changes associated with disease at both the cellular and subcellular level, such as cellular and nuclear enlargement and crowding and an increased nucleus-to-cytoplasm ratio [33].

\section{Clinical experience}

Pioneering work with DRS was conducted by Mourant et al. in 1996 [34]. Reflectance spectra were taken in vivo from the colon in 15 patients and from the stomach in 17 patients. The ratio of the area under the reflectance curve between 540 and $580 \mathrm{~nm}$ to that under the reflectance curve between 400 and $420 \mathrm{~nm}$ was used to discriminate neoplastic from non-neoplastic colon tissue. The slope of the spectrum in the 435- to 440-nm range was used to reasonably separate active colitis from quiescent colitis and normal colonic mucosa. Gastric dysplasia was found in only one case, and this was separated from normal and inflamed gastric epithelium by using the ratio between 355 and $360 \mathrm{~nm}$ to that between 630 and $635 \mathrm{~nm}$. When multiple linear regression was used for spectral classification, adenomatous polyps were distinguished from hyperplastic polyps with a sensitivity of $89 \%$ and a specificity of $75 \%$ [35].

Zonios et al. [2] collected diffuse reflectance spectra in vivo on adenomatous polyps and adjacent normal colon in 13 patients undergoing colonoscopy. Significant spectral differences were observed, especially around $420 \mathrm{~nm}$ (hemoglobin absorption valley), and these were attributed to the increased hemoglobin concentration (six times higher) of polyps relative to that of normal colon. In contrast, oxygen saturation was found to be similar in both normal and adenomatous tissue.

Wallace et al. [36] measured diffuse reflectance spectra in 13 patients with a diagnosis of Barrett's esophagus or suspected esophageal cancer. The sites measured were subsequently excised and submitted for pathologic assessment. The data were analyzed by a model in which a site was classified as dysplastic if more than $30 \%$ of the nuclei were enlarged, with enlarged defined as having a diameter greater than $10 \mu \mathrm{m}$. Based on this model, the samples were correctly classified with a high degree of sensitivity and specificity ( $90 \%$ and $90 \%$, respectively) for detecting the presence of either high grade or low grade dysplasia. 
In a study by Lovat et al. [3], DRS measurements were collected in vivo from 81 patients with high grade dysplasia or early cancer within Barrett's esophagus, then matched with histological specimens taken from identical sites within the segments of Barrett's esophagus. All biopsy specimens were reviewed by three gastrointestinal pathologists and classified as low risk (no dysplasia or low grade dysplasia) or high risk (high grade dysplasia or cancer). DRS detected high risk sites with a high sensitivity of $92 \%$, but the specificity was only $60 \%$. The specificity increased to $79 \%$ when ESS was used to discriminate high risk sites from areas of inflammation. In a concurrent study from the same group, conducted in 45 patients undergoing colonoscopy, ESS was able to discriminate with good accuracy among the different disease groups investigated, including adenomatous from hyperplastic polyps, cancer from adenomas, inflammatory bowel disease (IBD) from normal colon, and dysplastic polyps from IBD [37]. Low coherence enhanced backscattering (LEBS) spectroscopy, a variant of ESS, was recently proposed as an endoscopic prescreening method for colorectal cancer screening. Based on the concept of field carcinogenesis, micro-scale spectral changes were measured in biopsy specimens of endoscopically normal rectum obtained from 219 patients undergoing colonoscopy to predict whether their colon harbored cancer or advanced adenomas elsewhere [38]. LEBS-detectable alterations in endoscopically normal rectum were seen to mirror neoplasia progression in patients with no neoplasia, 5- to 9-mm adenomas, and advanced adenomas. For advanced adenomas, the LEBS signals had a sensitivity of $100 \%$ and a specificity of $80 \%$. Further studies with an endoscopically compatible fiberoptic probe are under way for validation of these results in vivo.

\section{Angle-resolved low coherence interferometry}

Changes in the density and size of cell nuclei are considered characteristic biomarkers of dysplasia. By analyzing the angular distribution of the backscattered light from a sample at different depths, this technique enables the measurement of cellular and nuclear morphology and offers the potential to assess cellular structure and organization in vivo.

Pyhtila et al. [39] developed a probe-based frequency-domain angle-resolved low coherence interferometry $(\mathrm{fa} / \mathrm{LCI}$ ) system with a subsecond acquisition time to make depth-resolved (up to $150 \mu \mathrm{m}$ ) quantitative measurements of changes in the size and texture of cell nuclei in tissue samples. Three esophageal specimens from three patients, who underwent esophagectomy for Barrett's neoplasia, were measured to examine the average nuclear size and density in healthy and dysplastic tissue, and the findings were in complete concordance with histology.

In vivo results [40] reported sensitivity as high as $100 \%$ and specificity of $84 \%$ for the discrimination of dysplasia with a fiber probe system for clinical fa/LCI (Oncoscope, Durham, North Carolina, USA) in 46 patients undergoing routine surveillance for Barrett's esophagus.

\section{Molecular imaging}

Endoscopic molecular imaging with fluorescently labeled targeted peptides is being increasingly investigated for the detection of inconspicuous dysplasia or cancer [41,42].

Ito et al. [43] used an anti-CEA antibody tagged with a novel fluorescent label (ICG-sulfo-OSu) and an infrared fluorescence endoscope to stain and image freshly resected specimens of gastric cancer from three patients. They found good correlation between the intensity of the infrared fluorescent signal and the cancerous sites, suggesting that this technique can spot cancer cells and generate a relatively strong fluorescent signal to detect small cancers.

Keller et al. [8] conducted an in vivo study in 27 patients with colonic polypoid lesions ( 8 adenomas, 25 carcinomas) by using a fluorescein-labeled antibody against CEA applied topically during conventional endoscopy. Fluorescence emission was visualized after 10 minutes of incubation with a standard fiberoptic endoscope modified with a 490-nm narrow-band filter positioned at the light source and a special lens containing a built-in 520-nm narrow-band filter housed in the attached camera. Although ulceration and bleeding proved to be limiting factors, this approach led to high specificity (100\%), modest sensitivity (78.6\%), and an overall diagnostic accuracy of $89.3 \%$.

An indocyanine green-labeled antibody against the epithelial membrane antigen (EMA) was developed by Muguruma et al. [44]. This compound exhibited a specific fluorescence emission at $807 \mathrm{~nm}$ under infrared excitation $(768 \mathrm{~nm})$ and was used to stain paraffin sections of normal esophagus.

The use of molecular biomarkers in combination with confocal endomicroscopy was first investigated by Hsiung et al. [45] to develop a fluorescent probe for detecting colon cancer. They identified a specific heptapeptide sequence (VRPMPLQ), which was conjugated with fluorescein and tested in patients undergoing colonoscopy. The fluorescein-conjugated peptide was administered topically and was found to bind more strongly to dysplastic cells than to adjacent normal cells with $81 \%$ sensitivity and $82 \%$ specificity.

Fluorescein isothiocyanate (FITC)-labeled anti-EGFR antibody and CLE were used to perform fluorescence targeting in mice with subcutaneous grafts of human colorectal cancer and in 15 endoscopic/surgical specimens of human colon tissue (six of them neoplastic) incubated in labeled EGFR antibody solution [10]. In the animal model, 45 minutes after injection of the antibodies, a specific fluorescent signal from the tumor was observed in vivo when a handheld rigid confocal probe (Optiscan, Notting Hill, Victoria, Australia) was placed in contact with the tissue with an excitation wavelength of $488 \mathrm{~nm}$ and a detection band range of 505 to $585 \mathrm{~nm}$. Interference from baseline tissue autofluorescence and nonspecific antibody binding was excluded by imaging the tumor before injection of the antibodies and after injection of a negative control. In the unfixed human tissue specimens, confocal imaging was performed mainly with the handheld probe and in two cases with a confocal endomicroscope (Pentax, Tokyo, Japan), which have characteristics in common. CLE analysis of EGFR expression, with histopathology used as the gold standard to classify the specimens, showed a statistically significant contrast $(P=0.0035)$ between neoplastic and nonneoplastic tissues, although weak fluorescence was also found in two samples of ischemia.

A similar study was performed by the same group [46] in ApcMin mice, xenograft models, and surgical specimens of patients with colorectal cancer. A vascular endothelial growth factor (VEGF)specific signal was visualized in vivo in 13 of 15 ApcMin mice, in 9 of 10 xenograft tumors, in 12 of 13 neoplastic specimens, and in 10 of 11 normal samples collected from patients during endoscopy. Both these studies advanced the concept of the feasibility of endoscopic molecular imaging in vivo.

Recently, AFI and a fluorescently labeled lectin, Tritiicum vulgare agglutinin (WGA), have been used to detect changes in glycan expression on the epithelial cell surface associated with the transition from Barrett's esophagus through dysplasia to adenocarcino- 
ma [47]. In particular, AFI was used to image WGA in four esophagectomy specimens obtained immediately after surgery. The specimens were intubated from the proximal end, and both white light and autofluorescence images were acquired at baseline. Fluorescein-labeled WGA was sprayed over the esophageal mucosa and imaged with 395- to 475-nm excitation. Specimens were then opened along their vertical axis and imaged with an IVIS camera (Caliper Life Sciences, Hopkinton, Massachusetts, USA) to enable the quantification of fluorescence and registration with histology. A highly significant statistical correlation between WGA fluorescence and degree of dysplasia was found $(P=$ 0.0002 ), with areas of high grade dysplasia and cancer showing lower fluorescence intensity and WGA binding relative to areas of nondysplastic Barrett's esophagus and normal esophageal mucosa.

The toxicity of molecular probes is a critical point to consider when molecular imaging is translated into the clinical setting. This issue could significantly impair and limit the clinical application of the method in vivo. Using concentrations below those known or shown to cause toxicity in animal models and for shorter periods of time is therefore paramount. Another point to consider is the way to deliver these agents. Local application through the endoscope, as in the study by Bird-Lieberman et al. [47], would be preferable to a systemic delivery method. Considerable research is currently under way to develop innovative nontoxic or less phototoxic drugs and delivery mechanisms for fluorescently labeled probes.

\section{Techniques for spatial resolution enhancement}

$\nabla$

\section{Optical coherence tomography}

OCT is the optical equivalent of ultrasound imaging, in which ultrasound waves are replaced by light. As with endoscopic ultrasound (EUS), two main types of OCT are available - linear and radial.

The basic components of an OCT system are a low coherence light source and a beam splitter, which splits the light into two identical beams, one directed to the tissue and the other to a mirror. An interferometer is used to combine the signals coming back from the tissue and the mirror; following signal-processing steps, cross-sectional images are obtained. Contrast in OCT is essentially due to reflected or simply backscattered light from refractive index mismatches within tissue.

OCT is a high speed (approximately 4 frames per second [48]) vertical plane imaging modality that combines confocal scanning capability with low coherence interferometry, resulting in excellent rejection of scattered light and deep tissue penetration. The use of near-infrared light (from approximately 800 to $2500 \mathrm{~nm}$ ) allows OCT to penetrate into scattering media more deeply (up to $4 \mathrm{~mm}$, depending on tissue type) than confocal microscopy, with typical lateral resolution of $15 \mu \mathrm{m}$ and axial resolution of $10 \mu \mathrm{m}$, although research systems have demonstrated axial resolution on the order of $1 \mu \mathrm{m}$ [48].

\section{Clinical experience}

Optical discrimination of the gastrointestinal tissue layers was first described in a landmark paper by Tearney et al. [49], who collected normal and diseased tissues and imaged them with OCT. Exploratory in vivo studies by Jackle et al., using portable OCT equipment and a fiberoptic flexible probe during routine endoscopic procedures, showed distinct OCT patterns in the mu- cosal structure of normal esophagus, reflux esophagitis, Barrett's esophagus and Barrett's esophagus-related adenocarcinoma [50], and colonic adenoma and adenocarcinoma [51]. Subsequently, Zuccaro et al. [52] showed that OCT could discriminate normal esophageal mucosa from cancer (both adenocarcinoma and squamous cell carcinoma).

Other studies further characterized differences in the structure of normal colon, adenomatous polyps, and hyperplastic polyps, with dysplasia outlined by a loss of tissue organization and reduced light scattering [53], and in the OCT patterns of normal colon, radiation proctitis, and adenocarcinoma [54]. OCT has been used in the detection of dysplasia in Barrett's esophagus. Modest sensitivity and specificity were reported in a prospective doubleblind study using a dual-channel endoscope (68\%, 82\%) [55], and in a study using an OCT image scoring system based on consensus histopathological diagnoses in the detection of high grade dysplasia and intramucosal carcinoma (83\%, 75\%) [56]. The role of endoscopic OCT in unmasking buried glands in Barrett's esophagus before and after radiofrequency ablation has also been described $[57,58]$.

This novel technique clearly has the intrinsic potential to reach the muscularis mucosae and thus discriminate noninvasive from invasive neoplasia. In a comparative study in normal tissue, Das et al. [59] showed that OCT has a higher resolution than high frequency $(20-30 \mathrm{MHz})$ EUS for imaging this layer with accuracy. Accurate delineation of the muscularis mucosae would in fact assist clinicians in determining whether endoscopic therapy rather than surgery should be recommended. In vivo endoscopic OCT was also used to assess the efficacy of radiofrequency ablation in treating persistent rectal bleeding from radiation proctitis unresponsive to conventional therapy [60].

A form of OCT, termed comprehensive volumetric microscopy, has been proposed that acquires a three-dimensional microscopic image of the full thickness of the distal esophagus [31, 32]. In a preliminary study by Suter et al. [61], volumetric microscopy of the distal esophagus was performed in 66 patients referred for Barrett's esophagus screening with a custom balloon catheter $(2.5 \mathrm{~cm}$ in diameter) that enabled spiral cross-sectional optical frequency domain imaging, a high speed second-generation embodiment of OCT. With histopathology used as the gold standard, optical frequency domain imaging showed increased sensitivity for the detection of specialized intestinal metaplasia in comparison with standard endoscopy.

Columnar epithelium and squamous epithelium in a region of heterotopic gastric mucosa (cervical inlet patch) in the upper esophagus were clearly distinguished when a through-the-scope three-dimensional OCT prototype probe was used. En face images were acquired to a depth of $400 \mu \mathrm{m}$ below the tissue surface and were compared with histology. The examination of biopsy specimens taken from the imaged lesion confirmed the endoscopic finding [62].

\section{Two-photon and multi-photon microscopy}

Biological tissues are, of course, inherently three-dimensional and typically have a high degree of scattering due to discontinuities in the refractive index and the heterogeneity of their constituents, which make high resolution three-dimensional imaging challenging.

Optically sectioned fluorescence imaging can be realized with single-photon confocal (visible light) or multi-photon excitation. In multi-photon microscopy (MPM), the excitation occurs through the absorption of multiple photons of lower energy (ty- 
pically halved in two-photon microscopy). No pinhole is required to prevent out-of-focus fluorescence from reaching the detector because excitation occurs only at the excitation spot. However, high incident power is required for multi-photon absorption to take place.

A major advantage of MPM is the longer wavelength of the light necessary to induce fluorescence (generally near-infrared, 700 $1000 \mathrm{~nm}$ ). Tissue absorption and scattering are limited with nearinfrared, allowing deep penetration imaging. A number of groups have developed miniaturized multi-photon microscopy endoscopes [63-65]. However, no commercially available multi-photon endoscope system is available to date.

Rivera et al. [66] demonstrated a compact flexible raster scanning microendoscope capable of MPM and second harmonic generation by imaging mouse tissue ex vivo at $800-\mathrm{nm}$ excitation. Images were acquired at 4.1 frames per second with a field of view of $110 \times 110 \mu \mathrm{m}$ when a double-clad fiber and a mode-locked Ti: sapphire laser were used as the excitation source. The distal end of the fiber was mounted onto a resonant/nonresonant scanner, which moved the fiber tip in a raster pattern across the back aperture of a miniaturized gradient index lens assembly, enabling two-dimensional lateral scanning.

In normal colon specimens excited at $735 \mathrm{~nm}$ with MPM, peak fluorescence emission intensity in the 410- to $490-\mathrm{nm}$ wavelength range has been reported [67]. It was suggested that this fluorescence at relatively longer wavelengths could be attributed to flavins and possibly lipofuscin, which are respectively found in cells within the interstitial space and leukocytes within the lamina propria.

In a study of freshly excised gastrointestinal tissue from 10 normal and 5 transgenic mice, excitation spectral signatures of epithelium, lamina propria, collagen, and lymphatic tissue were determined by using hyperspectral two-photon microscopy with $740-\mathrm{nm}$ excitation and three emission channels $(350-505 \mathrm{~nm}$, $505-560 \mathrm{~nm}$, and $560-650 \mathrm{~nm}$ ). Based on the three-dimensional sectioned two-photon images acquired, the morphology of these four main components was clearly visualized and compared with cross-sectional histology with a high degree of concordance [68]. Using MPM at 800-nm excitation, Zhuo et al. [69] performed a quantitative analysis of the redox ratio reduced nicotinamide adenine dinucleotide (NADH)/flavin adenine dinucleotide (FAD) to quantitatively monitor colonic cancer progression. The redox ratio was shown to increase with progression from dysplasia to cancer.

In a recent video and article from Schurmann et al. [70], fresh samples collected during endoscopy from controls and patients with IBD within 3 hours of excision were analyzed with singlecolor and multicolor imaging and spectral separation (395$415 \mathrm{~nm} / 435-465 \mathrm{~nm} / 540-580 \mathrm{~nm}$ ) at $800-\mathrm{nm}$ excitation. MPM made it possible to observe and compare tissue morphology in normal and inflamed specimens (from different patients) and to attribute the fluorescent signals detected to the different structures presumably identified (e.g., basal membrane, epithelial cells, and interstitial cells in the lamina propria). However, no statistically significant changes were reported, the normal interpatient variability was not characterized, and the correlation between fluorescent signals and tissue fluorophores and structures appeared to be too simplistic.

\section{Second and third harmonic generation imaging}

Like MPM, second harmonic generation (SHG) and third harmonic generation (THG) imaging depend on the simultaneous inter- action of two or three photons with the tissue under investigation. A long excitation wavelength, typically in the near-infrared range, is required for these phenomena to take place. However, whereas in MPM two photons are absorbed to produce a single fluorescent photon, in SHG and THG the photons are scattered, not absorbed by the tissue, to produce a new photon with two (SHG) or three (THG) times the energy of the incident photons. With these techniques, because of the absence of absorption, heating and damage of the sample are less likely to occur. Another advantage is the potential for three-dimensional imaging. SHG techniques allow the imaging of "non-centrosymmetric" structures, such as collagen, with reported tissue penetration depths between 100 and $300 \mu \mathrm{m}$ at laser excitation in the nearinfrared range. Therefore, they have been increasingly applied to characterize the structural changes occurring in the extracellular matrix during disease. An example is the desmoplastic reaction that typically accompanies the progression of many cancers. Using SHG microscopy at 800-nm excitation, Zhuo et al. [71] imaged 72 fresh colonic biopsy specimens from 32 patients and were able to quantify significant changes in the circle length of the crypt basement membranes (from en face SHG images) of normal, precancerous, and cancerous colonic tissues. Another important potential application of SHG microscopy is imaging fibrosis in liver and IBD.

\section{Coherent anti-Stokes Raman scattering microscopy}

Based on contrast derived from molecular vibration, CARS has the potential to provide fast imaging on a molecular level that is label free and has three-dimensional sectioning capability. Unlike Raman spectroscopy, CARS employs multiple photons to probe the molecular vibrations and produces a signal in which the emitted waves are coherent with one another. As a result, the CARS signal is orders of magnitude stronger than spontaneous Raman emission and so can provide much faster imaging. A detailed comparison of Raman imaging and CARS microscopy was carried out by Krafft et al. [72] in ex vivo colon tissue sections, raising the intriguing prospect of a combined complementary method for label-free live-cell imaging at video-rate speed, which is ideal for most clinical applications. Although prototype endoscopes capable of collecting CARS signals have been developed $[73,74]$, progress in delivering in vivo CARS imaging in gastrointestinal endoscopy is still awaited.

\section{Summary}

\section{$\nabla$}

Recent advances in gastrointestinal endoscopy constitute an unprecedented leap forward in basic and clinical research for both patients and clinicians. In theory, these advancements offer the yearned-for possibility of increasing the rates of detection of high risk lesions and the ability to target biopsies and resections, and so reduce the need for biopsy, costs, and uncertainty for both patients and clinicians. However, there appear to be two separate research communities, one working with sophisticated endoscopic technology and the other with disease biomarkers, functioning independently. It would seem sensible to integrate these two approaches to identify risk predictors and determine which modality is best for each patient population. All the emerging biophotonic techniques in endoscopy, with their pros and cons and clinical experience to date, are summarized in $\bullet$ Table 1.

It is still unknown which technique or combination of techniques offers the best potential. The optimal method will probably have 
the ability to survey wide areas of tissue in concert with the ability to obtain the necessary degree of detailed information provided by point measurements. This would be made possible by coupling a wide-field modality to a mode of single-point measurement.

One optical technology potentially capable of meeting these requirements is FLIM. Nevertheless, to date FLIM has been investigated in the gastrointestinal tract only ex vivo in a relatively small number of samples, and further studies are required to confirm the initial findings. Systematic studies in vivo of the fluorescence lifetime signatures in normal and diseased tissue are crucial for acquiring a complete understanding of the origin of the autofluorescence contrast observed, and for assessing the ability of fluorescence lifetime technology to provide an accurate clinical diagnosis.

Techniques such as MPM, SHG, and CARS have not yet made the transition between research and in vivo clinical application. However, the diagnostic morphologic-functional capability of MPM is superlative, and in the near future this technique is likely to be the winner.

Raman spectroscopy offers the exciting prospect of revealing highly specific biochemical changes before morphologic differences between normal and diseased tissue become detectable. However, it cannot be readily extended to provide a wide-field screening tool that can image fields of view of several centimeters.

Among all the biophotonic techniques described in this review, OCT is certainly the most mature. OCT has brought optical resolution down to a few microns, enabling for the first time three-dimensional tissue microscopy in vivo during endoscopy. However, although of invaluable assistance for tissue characterization, this technique can only infer structural aspects and does not provide functional information about tissue. Thus, rather than in detection or screening, this technique may have a role in determining prognosis and predicting treatment response.

Because of the highly accurate detection of early cytological changes, DRS may have a role as a prescreening method before conventional cancer screening endoscopy to rule out the presence of a field effect and therefore identify patients potentially harboring neoplasia.

Lastly, molecular imaging represents the quintessence of the application of biophotonics to endoscopy, in that molecular biomarkers and fluorescence detection are exploited for targeted diagnosis at the cellular and subcellular levels. In contrast with tissue autofluorescence alone, in which the fluorescence signal is often weak, labeled antibodies can provide highly specific contrast. However, they must be certified for human use, so that course assessments for safety and pharmacokinetics are required, and this clearly limits their immediate clinical utility.

Crucial to the clinical translation of all these techniques to endoscopy is the development of compact and real-time systems that are suitable for in vivo clinical applications. For instance, since the end of the $1990 \mathrm{~s}$, several FLIM endoscope designs have been proposed [75-82], and current efforts are directed to implement and validate this technology for modern endoscopy.

In fluorescence-based techniques, the greatest technological barrier to overcome is the background fluorescence from the fiber probes. At the wavelengths typically used for autofluorescence studies (310 and $420 \mathrm{~nm}$ ) [83,84], the fluorescence from the fibers occurs over the whole length of the fiber bundle, is very strong, and completely masks the fluorescence arising from the area of tissue under investigation. The limit is fixed at $420 \mathrm{~nm}$, the shortest excitation wavelength currently exploitable with this implementation. One possible way to overcome the limitation is to construct fiber prototypes with lower background fluorescence so as to avoid the unwanted background fluorescence exhibited by the fiber bundle used to carry the excitation light.

Another important limitation of these techniques is that it is not known whether the proposed exposure to light (e.g., ultraviolet A) delivered to the gastrointestinal mucosal epithelium during endoscopy is safe. Strategies to overcome this limitation include restricting the average optical power at the sample in order to minimize the risk for tissue damage. There is currently no accepted limit on the exposure of internal tissues to light. In the absence of a suitable standard, the International Commission on Non-Ionizing Radiation Protection (ICNIRP) guidelines regarding skin exposure are the most appropriate ones to follow. Irradiance at power levels lower than the maximum permissible exposure (MPE) for skin is therefore recommended at present. Previous work in the literature indicates that these levels of exposure caused no increase in triphosphate-biotin nick end-labeling (TUNEL)-positive cells above baseline compared with controls in porcine esophagus and colon [85]. However, further studies of phototoxicity and in vivo applications are required to identify the most discriminating wavelengths and lifetimes in order to create a relevant diagnostic model to demonstrate performance. Other challenges are the following: (i) to develop suitable compact, portable systems that can be housed in a way that is appropriate for clinical use, allowing them to be easily sterilized and transported; (ii) to develop software tools for the analysis and interpretation of data; and (iii) to apply the instrumentation to patients. Essential for the transition of photonic approaches into clinical diagnostics is therefore the contribution of clinicians in designing, guiding, and assessing the specification, sterilization, ethical approval, and clinical use of these techniques.

Whether these techniques will soon be useful in clinical practice is still under investigation. Further validation studies are urgently awaited, and several groups worldwide are currently working toward this milestone.

\section{Competing interests: None}

\section{Institutions}

${ }^{1}$ Section of Gastroenterology and Hepatology, Department of Medicine, Imperial College London, London, United Kingdom

2 Photonics Group, Department of Physics, Imperial College London, London, United Kingdom

3 Endoscopy Unit, Department of Gastroenterology, Charing Cross Hospital, Imperial College Healthcare NHS Trust, London, United Kingdom

${ }^{4}$ Department of Endoscopy, North East London NHS Treatment Centre, Care UK, London, United Kingdom

${ }^{5}$ Department of Gastroenterology and Hepatology, University Medical Center Utrecht, Utrecht, The Netherlands

${ }^{6}$ Experimental Histopathology Laboratory, Cancer Research UK London Research Institute, London, United Kingdom

${ }^{7}$ Department of Histopathology, Imperial College London, London, United Kingdom 


\section{Acknowledgment \\ $\nabla$}

This work was supported by the UK Engineering and Physical Sciences Research Council (EPSRC, grant reference: EP/F040202/1). Dr. Sergio Coda acknowledges an EPSRC doctoral prize fellowship (EP/K502856/1). The authors gratefully thank Professor Paul French and Dr. Chris Dunsby of the Photonics Group, Department of Physics, at Imperial College London for their invaluable supervision, advice, and support.

\section{References}

1 Coda S, Thillainayagam AV. State of the art in advanced endoscopic imaging for the detection and evaluation of dysplasia and early cancer of the gastrointestinal tract. Clin Exp Gastroenterol 2014; 7: 133 - 150

2 Zonios G, Perelman LT, Backman V et al. Diffuse reflectance spectroscopy of human adenomatous colon polyps in vivo. Appl Opt 1999; 38: $6628-6637$

3 Lovat LB, Johnson K, Mackenzie GD et al. Elastic scattering spectroscopy accurately detects high grade dysplasia and cancer in Barrett's oesophagus. Gut 2006; 55: 1078 - 1083

4 Georgakoudi I, Jacobson BC, Van Dam J et al. Fluorescence, reflectance, and light-scattering spectroscopy for evaluating dysplasia in patients with Barrett's esophagus. Gastroenterology 2001; 120: 1620-1629

5 Shao $X$, Zheng $W$, Huang $Z$. Polarized near-infrared autofluorescence imaging combined with near-infrared diffuse reflectance imaging for improving colonic cancer detection. Opt Express 2010; 18: 24293 24300

6 Marcu L, French PMW, Elson DS. Fluorescence lifetime spectroscopy and imaging: principles and applications in biomedical diagnostics. Philadelphia, PA: Taylor \& Francis; 2014

7 McGinty J, Galletly NP, Dunsby C et al. Wide-field fluorescence lifetime imaging of cancer. Biomed Opt Express 2010; 1: 627-640

8 Keller R, Winde G, Terpe HJ et al. Fluorescence endoscopy using a fluorescein-labeled monoclonal antibody against carcinoembryonic antigen in patients with colorectal carcinoma and adenoma. Endoscopy 2002; 34: $801-807$

9 Mayinger B, Neumann F, Kastner $C$ et al. Early detection of premalignant conditions in the colon by fluorescence endoscopy using local sensitization with hexaminolevulinate. Endoscopy 2008; 40: 106-109

10 Goetz M, Ziebart A, Foersch S et al. In vivo molecular imaging of colorectal cancer with confocal endomicroscopy by targeting epidermal growth factor receptor. Gastroenterology 2010; 138: 435-446

11 Mycek M, Schomacker K, Nishioka N. Colonic polyp differentiation using time-resolved autofluorescence spectroscopy. Gastrointest Endosc 1998; 48: 390-394

12 Pfefer TJ, Paithankar DY, Poneros JM et al. Temporally and spectrally resolved fluorescence spectroscopy for the detection of high grade dysplasia in Barrett's esophagus. Lasers Surg Med 2003; 32: 10-16

13 Chen HM, Chiang CP, You C et al. Time-resolved autofluorescence spectroscopy for classifying normal and premalignant oral tissues. Lasers Surg Med 2005; 37: 37-45

14 Thompson AJ, Coda S, Brydegaard Sørensen $M$ et al. In vivo measurements of diffuse reflectance and time-resolved autofluorescence emission spectra of basal cell carcinomas. J Biophotonics 2012; 5: 240 - 254

15 Coda S, Thompson AJ, Kennedy GT et al. Fluorescence lifetime spectroscopy of tissue autofluorescence in normal and diseased colon measured ex vivo using a fiber-optic probe. Biomed Opt Express 2014; 5: $515-538$

16 Skala MC, Riching KM, Bird DK et al. In vivo multiphoton fluorescence lifetime imaging of protein-bound and free nicotinamide adenine dinucleotide in normal and precancerous epithelia. J Biomed Opt 2007; 12: 024014

17 Chang CL, You C, Chen HM et al. Autofluorescence lifetime measurement on oral carcinogenesis. Conf Proc IEEE Eng Med Biol Soc 2004; 4: $2349-2351$

18 Li B, Zhang Z, Xie S. Steady state and time-resolved autofluorescence studies of human colonic tissues. Chin Opt Lett 2006; 4: 348-350

19 Sun Y, Phipps JE, Meier J et al. Endoscopic fluorescence lifetime imaging for in vivo intraoperative diagnosis of oral carcinoma. Microsc Microanal 2013; 19: $791-798$

20 Shim MG, Song LM, Marcon NE et al. In vivo near-infrared Raman spectroscopy: demonstration of feasibility during clinical gastrointestinal endoscopy. Photochem Photobiol 2000; 72: 146-150
21 Molckovsky A, Song LM, Shim MG et al. Diagnostic potential of near-infrared Raman spectroscopy in the colon: differentiating adenomatous from hyperplastic polyps. Gastrointest Endosc 2003; 57: 396-402

22 Stone $N$, Kendall C, Shepherd $N$ et al. Near-infrared Raman spectroscopy for the classification of epithelial pre-cancers and cancers. J Raman Spectrosc 2002; 33: 564-573

23 Day JC, Bennett R, Smith B et al. A miniature confocal Raman probe for endoscopic use. Physics Med Biol 2009; 54: 7077 - 7087

24 Kendall C, Day J, Hutchings J et al. Evaluation of Raman probe for oesophageal cancer diagnostics. Analyst 2010; 135: 3038 - 3041

25 Almond LM, Hutchings J, Kendall C et al. Assessment of a custom-built Raman spectroscopic probe for diagnosis of early oesophageal neoplasia. J Biomed Opt 2012; 17: 081421 - 081421

26 Almond LM, Hutchings J, Lloyd G et al. Endoscopic Raman spectroscopy enables objective diagnosis of dysplasia in Barrett's esophagus. Gastrointest Endosc 2014; 79: 37-45

27 Bergholt MS, Zheng W, Lin K et al. In vivo diagnosis of esophageal cancer using image-guided Raman endoscopy and biomolecular modeling. Technol Cancer Res Treat 2011; 10: 103-112

28 Duraipandian S, Bergholt MS, Zheng $W$ et al. Real-time Raman spectroscopy for in vivo, online gastric cancer diagnosis during clinical endoscopic examination. J Biomed Opt 2012; 17: 081418

29 Shao $X$, Zheng $W$, Huang $Z$. Near-infrared autofluorescence spectroscopy for in vivo identification of hyperplastic and adenomatous polyps in the colon. Biosens Bioelectron 2011; 30: 118-122

30 Zhang G, Demos SG, Alfano RR. Far-red and NIR spectral wing emission from tissues under 532 and $632 \mathrm{~nm}$ photo-excitation. Lasers Life Sci 1999; 9: 1-16

31 Wagnieres GA, Star WM, Wilson BC. In vivo fluorescence spectroscopy and imaging for oncological applications. Photochem Photobiol 1998; 68: $603-632$

32 Wong Kee Song LM, Marcon NE. Fluorescence and Raman spectroscopy. Gastrointest Endosc Clin N Am 2003; 13: 279-296

33 Johansson A, Kromer K, Sroka R et al. Clinical optical diagnostics - status and perspectives. Med Laser Appl 2008; 23: 155-174

34 Mourant JR, Bigio IJ, Boyer JD et al. Elastic scattering spectroscopy as a diagnostic tool for differentiating pathologies in the gastrointestinal tract: preliminary testing. J Biomed Opt 1996; 1: $192-199$

$35 \mathrm{Ge} Z$, Schomacker KT, Nishioka NS. Identification of colonic dysplasia and neoplasia by diffuse reflectance spectroscopy and pattern recognition techniques. Appl Spectrosc 1998; 52: $833-839$

36 Wallace MB, Perelman LT, Backman Vet al. Endoscopic detection of dysplasia in patients with Barrett's esophagus using light-scattering spectroscopy. Gastroenterology 2000; 119: 677-682

37 Dhar A, Johnson KS, Novelli MR et al. Elastic scattering spectroscopy for the diagnosis of colonic lesions: initial results of a novel optical biopsy technique. Gastrointest Endosc 2006; 63: 257-261

38 Roy HK, Gomes A, Turzhitsky Vet al. Spectroscopic microvascular blood detection from the endoscopically normal colonic mucosa: biomarker for neoplasia risk. Gastroenterology 2008; 135: 1069-1078

39 Pyhtila JW, Chalut KJ, Boyer JD et al. In situ detection of nuclear atypia in Barrett's esophagus by using angle-resolved low-coherence interferometry. Gastrointest Endosc 2007; 65: 487-491

40 Terry NG, Zhu Y, Rinehart MT et al. Detection of dysplasia in Barrett's esophagus with in vivo depth-resolved nuclear morphology measurements. Gastroenterology 2011; 140: $42-50$

41 Goetz M, Wang TD. Molecular imaging in gastrointestinal endoscopy. Gastroenterology 2010; 138: 828-833.e821

42 Weissleder R, Ross BD, Rehemtulla $A$ et al. Molecular imaging: principles and practice. Beijing, China: People's Medical Publishing House; 2010

43 Ito S, Muguruma N, Kusaka Yet al. Detection of human gastric cancer in resected specimens using a novel infrared fluorescent anti-human carcinoembryonic antigen antibody with an infrared fluorescence endoscope in vitro. Endoscopy 2001; 33: 849-853

44 Muguruma N, Ito S, Hayashi S et al. Antibodies labeled with fluorescence-agent excitable by infrared rays. J Gastroenterol 1998; 33: 467-471

45 Hsiung P-L, Hardy J, Friedland $S$ et al. Detection of colonic dysplasia in vivo using a targeted heptapeptide and confocal microendoscopy. Nat Med 2008; 14: 454-458

46 Foersch S, Kiesslich R, Waldner MJ et al. Molecular imaging of VEGF in gastrointestinal cancer in vivo using confocal laser endomicroscopy. Gut 2010; 59: $1046-1055$ 
47 Bird-Lieberman EL, Neves AA, Lao-Sirieix $P$ et al. Molecular imaging using fluorescent lectins permits rapid endoscopic identification of dysplasia in Barrett's esophagus. Nat Med 2012; 18: 315-321

48 Tearney GJ, Brezinski ME, Bouma BE et al. In vivo endoscopic optical biopsy with optical coherence tomography. Science 1997; 276: 2037-2039

49 Tearney GJ, Brezinski ME, Southern JF et al. Optical biopsy in human gastrointestinal tissue using optical coherence tomography. Am J Gastroenterol 1997; 92: $1800-1804$

50 Jackle S, Gladkova N, Feldchtein F et al. In vivo endoscopic optical coherence tomography of esophagitis, Barrett's esophagus, and adenocarcinoma of the esophagus. Endoscopy 2000; 32: 750-755

51 Jackle S, Gladkova N, Feldchtein F et al. In vivo endoscopic optical coherence tomography of the human gastrointestinal tract - toward optical biopsy. Endoscopy 2000; 32: $743-749$

52 Zuccaro G, Gladkova N, Vargo J et al. Optical coherence tomography of the esophagus and proximal stomach in health and disease. Am J Gastroenterol 2001; 96: 2633-2639

53 Pfau PR, Sivak MVJr, Chak A et al. Criteria for the diagnosis of dysplasia by endoscopic optical coherence tomography. Gastrointest Endosc 2003; 58: 196-202

54 Adler DC, Zhou C, Tsai TH et al. Three-dimensional endomicroscopy of the human colon using optical coherence tomography. Opt Express 2009; 17 : 784-796

55 Isenberg G, Sivak MVJr, Chak A et al. Accuracy of endoscopic optical coherence tomography in the detection of dysplasia in Barrett's esophagus: a prospective, double-blinded study. Gastrointest Endosc 2005; 62: $825-831$

56 Evans JA, Poneros JM, Bouma BE et al. Optical coherence tomography to identify intramucosal carcinoma and high-grade dysplasia in Barrett's esophagus. Clin Gastroenterol Hepatol 2006; 4: 38-43

57 Adler DC, Zhou C, Tsai TH et al. Three-dimensional optical coherence tomography of Barrett's esophagus and buried glands beneath neosquamous epithelium following radiofrequency ablation. Endoscopy 2009; 41: $773-776$

58 Zhou C, Tsai TH, Lee HC et al. Characterization of buried glands before and after radiofrequency ablation by using 3-dimensional optical coherence tomography (with videos). Gastrointest Endosc 2012; 76: $32-40$

59 Das A, Sivak MV, Chak A et al. High-resolution endoscopic imaging of the GI tract: a comparative study of optical coherence tomography versus high-frequency catheter probe EUS. Gastrointest Endosc 2001; 54 : 219-224

60 Zhou C, Adler DC, Becker $L$ et al. Effective treatment of chronic radiation proctitis using radiofrequency ablation. Therap Adv Gastroenterol 2009; 2: 149-156

61 Suter MJ, Nishioka NS, Yoo H et al. S1591: Barrett's esophagus screening using balloon-based optical frequency domain imaging: a comparison with endoscopy. Gastrointest Endosc 2010; 71: AB202

62 Zhou C, Kirtane T, Tsai TH et al. Cervical inlet patch-optical coherence tomography imaging and clinical significance. World J Gastroenterol 2012; 18: 2502-2510

63 Myaing MT, MacDonald DJ, Li X. Fiber-optic scanning two-photon fluorescence endoscope. Opt Lett 2006; 31: 1076-1078

64 Engelbrecht CJ, Johnston RS, Seibel EJ et al. Ultra-compact fiber-optic two-photon microscope for functional fluorescence imaging in vivo. Opt Express 2008; 16: $5556-5564$

65 Tang S, Jung W, McCormick D et al. Design and implementation of fiberbased multiphoton endoscopy with microelectromechanical systems scanning. J Biomed Opt 2009; 14: 034005
66 Rivera DR, Brown CM, Ouzounov DG et al. Compact and flexible raster scanning multiphoton endoscope capable of imaging unstained tissue. Proc Natl Acad Sci U S A 2011; 108: 17598-17603

67 Rogart JN, Nagata J, Loeser CS et al. Multiphoton imaging can be used for microscopic examination of intact human gastrointestinal mucosa ex vivo. Clin Gastroenterol Hepatol 2008; 6: 95-101

68 Grosberg LE, Radosevich AJ, Asfaha S et al. Spectral characterization and unmixing of intrinsic contrast in intact normal and diseased gastric tissues using hyperspectral two-photon microscopy. PLoS One 2011; 6: e19925

69 Zhuo S, Yan J, Chen $G$ et al. Label-free monitoring of colonic cancer progression using multiphoton microscopy. Biomed Opt Express 2011; 2: 615-619

70 Schurmann S, Foersch S, Atreya R et al. Label-free imaging of inflammatory bowel disease using multiphoton microscopy. Gastroenterology 2013; $145:$ 514-516

71 Zhuo S, Yan J, Chen G et al. Label-free imaging of basement membranes differentiates normal, precancerous, and cancerous colonic tissues by second-harmonic generation microscopy. PLoS ONE 2012; 7: e38655

72 Krafft C, Ramoji AA, Bielecki C et al. A comparative Raman and CARS imaging study of colon tissue. J Biophotonics 2009; 2: 303-312

73 Légaré F, Evans CL, Ganikhanov F et al. Towards CARS endoscopy. Opt Express 2006; 14: 4427-4432

74 Wang Z, Liu Y, Gao $L$ et al. Use of multimode optical fibers for fiberbased coherent anti-Stokes Raman scattering microendoscopy imaging. Opt Lett 2011; 36: 2967-2969

75 Mizeret J, Stepinac T, Hansroul M et al. Instrumentation for real-time fluorescence lifetime imaging in endoscopy. Rev Sci Instrum 1999; 70: $4689-4701$

76 Requejo-Isidro J, McGinty J, Munro I et al. High-speed wide-field timegated endoscopic fluorescence-lifetime imaging. Opt Lett 2004; 29: $2249-2251$

77 Munro I, McGinty J, Galletly N et al. Toward the clinical application of time-domain fluorescence lifetime imaging. J Biomed Opt 2005; 10: 051403

78 Elson DS, Jo JA, Marcu L. Miniaturized side-viewing imaging probe for fluorescence lifetime imaging (FLIM): validation with fluorescence dyes, tissue structural proteins and tissue specimens. New J Physics 2007; 9: 127

79 Kennedy GT, Manning HB, Elson DS et al. A fluorescence lifetime imaging scanning confocal endomicroscope. J Biophotonics 2010; 3: $103-107$

80 Fruhwirth GO, Ameer-Beg S, Cook R et al. Fluorescence lifetime endoscopy using TCSPC for the measurement of FRET in live cells. Opt Express 2010; 18: $11148-11158$

81 Cheng S, Rico-Jimenez IJ, Jabbour J et al. Flexible endoscope for continuous in vivo multispectral fluorescence lifetime imaging. Opt Lett 2013; 38: $1515-1517$

82 Sun Y, Phipps J, Elson DS et al. Fluorescence lifetime imaging microscopy: in vivo application to diagnosis of oral carcinoma. Opt Lett 2009; 34: $2081-2083$

83 Richards-Kortum R, Sevick-Muraca E. Quantitative optical spectroscopy for tissue diagnosis. Annu Rev Phys Chem 1996; 47: 555-606

84 Ramanujam $N$. Fluorescence spectroscopy of neoplastic and non-neoplastic tissues. Neoplasia 2000; 2: 89-117

85 Brozyna A, Chwirot BW. Different susceptibility of cells of porcine skin and internal organs to ultraviolet A-induced breaking of nuclear DNA. Photochem Photobiol 2005; 81: 674-681 